\title{
La valoración antropométrica en el contexto de la escuela como medida para detectar y prevenir efectos a largo plazo de la obesidad y del sobrepeso en niños en edad escolar
}

\author{
Amelia Fernández-Juan ${ }^{\mathrm{a}, *}$, Carolina Ramírez-Gil ${ }^{\mathrm{b}}$ y Laura van der Werf ${ }^{\mathrm{a}}$ \\ a Departamento de Medicina Preventiva, Facultad de Medicina, Pontificia Universidad Javeriana, Bogotá, Colombia \\ ${ }^{\mathrm{b}}$ Hospital Universitario de San Ignacio, Bogotá, Colombia
}

Recibido el 30 de junio de 2015; aceptado el 23 de junio de 2016

Disponible en Internet el 3 de septiembre de 2016

\section{PALABRAS CLAVE \\ Obesidad; \\ Prevención; \\ Riesgo de \\ enfermedad \\ coronaria}

\begin{abstract}
Resumen
Objetivo: El sobrepeso y la obesidad son un problema de salud pública de particular importancia en el contexto de las poblaciones en situación de mayor vulnerabilidad. Su importancia radica en el efecto de la obesidad en la infancia, sobre la salud cardiovascular futura. El objetivo de esta investigación fue describir la prevalencia del sobrepeso y la obesidad en la edad escolar en la población vulnerable en Bogotá y el contexto escolar en el cual se expresan.

Materiales y métodos: Se realizó una evaluación antropométrica en dos colegios. Se obtuvieron los Z score de la talla para la edad y el índice de masa corporal de acuerdo a los estándares de crecimiento de la Organización Mundial de la Salud y de los Centros para el Control y la Prevención de Enfermedades en Atlanta. Posteriormente se caracterizaron ambos colegios y se realizó un grupo focal con los profesores sobre lo que debe hacer parte de una escuela saludable.

Resultados y conclusiones: La prevalencia de la obesidad fue de 5,54\% en uno de los colegios y $3,68 \%$ en el otro. La prevalencia del sobrepeso fue de $14,61 \%$ en un colegio y $9,82 \%$ en el otro. A pesar de que la alimentación saludable fue identificada por los profesores como una característica importante de las escuelas, esto no se vio reflejado en el proyecto educativo institucional. Existen diferencias en cuanto a la prevalencia de la obesidad y del sobrepeso entre los colegios y entre los sexos, por lo cual existe la necesidad de continuar generando otras investigaciones que posibiliten indagar asociaciones causales.

(c) 2016 Sociedad Colombiana de Cardiología y Cirugía Cardiovascular. Publicado por Elsevier España, S.L.U. Este es un artículo Open Access bajo la licencia CC BY-NC-ND (http:// creativecommons.org/licenses/by-nc-nd/4.0/).
\end{abstract}

\footnotetext{
* Autor para correspondencia.

Correos electrónicos: afernand@javeriana.edu.co, afernand7@gmail.com (A. Fernández-Juan).
} 


\section{KEYWORDS}

Obesity;

Prevention;

Risk of coronary

heart disease

\section{Anthropometric assessment in the school context as a means for the detection and prevention of the long-term effects of obesity and over weight in children of school age}

\begin{abstract}
Objective: Overweight and obesity are a public health problem of particular importance for the most vulnerable populations. The problem is significant because of the impact of childhood obesity on future cardiovascular health. The objective of this investigation was to describe the prevalence of school- age overweight and obesity in Bogota's vulnerable population, and the school context in which they present.

Materials and methods: an anthropometric assessment was performed in two schools. The height for age and body mass index $\mathrm{Z}$ scores were obtained according to the growth standards of the World Health Organisation and the Centres for Disease Control and Prevention in Atlanta. Subsequently both schools were identified and a focus group was formed with the teachers regarding what a healthy school should comprise.

Results and conclusions: There was a 5.54\% prevalence of obesity in one of the schools, and $3.68 \%$ in the other. The prevalence of overweight was $14.61 \%$ in one school, and $9.82 \%$ in the other. Despite the fact that a healthy diet was highlighted by the teachers as an important characteristic in schools, this was not reflected in the institutional education project. There were differences in the prevalence of obesity and overweight between the schools, and between the sexes. Therefore, further studies are required to enable an in-depth exploration of the causative associations.

(c) 2016 Sociedad Colombiana de Cardiología y Cirugía Cardiovascular. Published by Elsevier España, S.L.U. This is an open access article under the CC BY-NC-ND license (http:// creativecommons.org/licenses/by-nc-nd/4.0/).
\end{abstract}

\section{Introducción}

El bienestar integral de todos los niños y las niñas ha sido una de las tareas pendientes del siglo XXI en muchas regiones del mundo. En este contexto, garantizar que los niños puedan alcanzar un crecimiento y desarrollo saludable, es generar oportunidades durante la infancia para que cada niño y cada niña, cuente con un potencial central, que relacionado con otras condiciones de vida, le sea útil para ubicarse en una ruta hacia una vida digna, superando los mínimos exigibles ${ }^{1}$.

El sobrepeso y la obesidad son un problema de salud pública de particular importancia en el contexto de las poblaciones en situación de mayor vulnerabilidad. Según un análisis secundario de la encuesta nacional de la situación nutricional en Colombia (ENSIN 2010), la obesidad continúa presentando mayor prevalencia en las poblaciones con mayor riqueza, no obstante, la incidencia de la obesidad es mayor en las poblaciones con un nivel socioeconómico más bajo que habitan en las zonas urbanas ${ }^{2}$.

La obesidad es, en estricto sentido, el exceso del tejido adiposo. En tanto los métodos para medir directamente el exceso del tejido adiposo no son de fácil uso en la práctica clínica diaria, se utilizan las medidas antropométricas, como medida indirecta para el tamizaje nutricional ${ }^{3}$.

Ahora bien, la definición de sobrepeso y obesidad varían de acuerdo al sistema de clasificación utilizado ${ }^{4}$, en los adultos, definir los puntos de corte para determinar a partir de qué índice de masa corporal se considera sobrepeso u obesidad, es más sencillo que en el caso de los niños. Esto se debe a que la definición en el caso de los adultos deriva del índice de masa corporal a partir del cual, la morbilidad aumenta de forma muy significativa ${ }^{5}$. En el caso de los niños, hay dificultades para ubicar estos puntos de corte, por cuanto los efectos de la obesidad infantil en la salud, en el corto plazo son menores que los observados en los adultos y, además, los riesgos para la salud están posiblemente mediados a través de la obesidad adulta ${ }^{5}$.

El efecto de la obesidad en la edad pediátrica, sobre la salud cardiovascular futura de las personas que la padecen no es del todo claro. A pesar de que se ha encontrado una relación entre la obesidad infantil y la enfermedad cardiovascular adulta, se ha observado que esta relación podría no ser directa; la asociación depende del índice de masa corporal en la adultez $z^{6,7}$. Existe una clara relación entre la obesidad infantil y la obesidad durante la adultez. En el estudio del riesgo cardiovascular en jóvenes en Finlandia se encontró que aquellos niños entre 3 y 9 años de edad, que se encontraban por encima del percentil 80 , tenían 3 veces más riesgo de tener un índice de masa corporal mayor de $30 \mathrm{~kg} / \mathrm{m}^{2}$ en la adultez ${ }^{6}$. Se ha observado que los niños obesos o con sobrepeso, obesos posteriormente durante la adultez tienen mayor riesgo de: la diabetes tipo 2, la hipertensión, la dislipidemia y la aterosclerosis de la carótida. Sin embargo, el riesgo para estos desenlaces en los niños obesos o con sobrepeso, que durante su adultez dejaron de ser obesos parecen ser similares a los de aquellos que nunca fueron obesos durante la infancia ${ }^{8}$. Además, se ha encontrado una asociación entre un índice de masa corporal para la edad clasificado por encima del percentil 99 de acuerdo a la clasificación del índice de masa corporal para la edad del CDC, con anomalías en la bioquímica sanguínea y un mayor riesgo de obesidad severa en la adulte $z^{9}$. Se ha 
descrito que tanto en los niños clasificados con sobrepeso como en los obesos, existe una mayor prevalencia de alteraciones relacionadas con el síndrome metabólico ${ }^{10,11}$. La obesidad y el sobrepeso en los niños y los adolescentes, son los principales factores de riesgo para el desarrollo de la diabetes mellitus tipo 2 juvenil $^{12}$.

En cuanto a las características del ambiente escolar, se ha encontrado que la presencia de tiendas de comida rápida se encuentra asociada a los hábitos alimentarios irregulares y al sobrepeso en los adolescentes, particularmente, en las poblaciones con bajo nivel socioeconómico ${ }^{13-15}$.

En este sentido y reconociendo la necesidad de realizar un tamizaje adecuado de la obesidad y el sobrepeso en los niños habitantes de las localidades más vulnerables de las grandes ciudades, iniciamos la evaluación antropométrica de los niños y las niñas matriculadas en dos colegios localizados en un territorio con predominio de barrios de origen informal y de alto conflicto social, ubicado en la localidad de Ciudad Bolívar en la ciudad de Bogotá.

Evaluamos el peso y la talla de los niños y las niñas matriculados desde kínder hasta quinto grado, con edades comprendidas entre los 5 y los 13 años de edad. Adicionalmente, caracterizamos los colegios y el contexto comunitario en el cual se encuentran inscritas.

\section{Antecedentes}

Ciudad Bolívar es una localidad que se encuentra ubicada en el suroccidente de la ciudad de Bogotá. Entre las veinte localidades que conforman Bogotá, es la cuarta localidad con mayor población y la tercera localidad con mayor extensión. Está conformada por 252 barrios legales y más de 100 barrios ilegales. La población de la localidad está en su mayoría conformada por: personas migrantes, reinsertadas, desmovilizadas o víctimas del conflicto provenientes de diferentes lugares del país, especialmente de: Tolima, Chocó, Meta y el nordeste antioqueño ${ }^{16}$.

Según diagnósticos realizados por el equipo del Hospital de Vista Hermosa, ubicado en la localidad, uno de cada 6 niños y adolescentes presentaba sobrepeso u obesidad; esta relación aumentaba a medida que se exploraban niños y niñas de estratos socioeconómicos más altos y a medida que el nivel educativo de la madre era más alto $(9,4 \%$ en madres sin educación vs. $26,8 \%$ en madres con educación superior $)^{16}$.

Según los datos obtenidos del sistema de vigilancia alimentaria y nutricional, el escolar colombiano promedio consume carbohidratos y azúcares en la jornada escolar, porque son los alimentos más económicos y de fácil acceso disponibles en las tiendas escolares. En contraparte, el consumo de frutas y proteínas es más bajo en esta población en los mismos escenarios ${ }^{17}$.

Ante esta situación, los organismos internacionales han empezado a proponer distintas políticas para lograr intervenciones eficaces en la educación nutricional y hábitos saludables de la niñez en la edad escolar primaria ${ }^{18,19}$.

En este ámbito, la escuela debe ser considerada un escenario fundamental para fortalecer la salud de la población en edad escolar. Se ha demostrado que tanto en países desarrollados como en países en desarrollo, los programas escolares pueden reducir simultáneamente los problemas de salud comunes, aumentar la eficiencia del sistema educativo y mejorar la salud pública, la educación y el desarrollo social y económico de cada nación ${ }^{20}$. La Organización Mundial de la Salud considera que una escuela promotora de la salud es aquella que refuerza constantemente su capacidad de actuar como entorno saludable para vivir, aprender y trabajar ${ }^{20,21}$. Dentro del contexto de la Escuela Saludable, se han realizado estudios para evaluar el impacto de intervenciones para mejorar las condiciones nutricionales de los niños en edad escolar. Se ha encontrado en diferentes estudios realizados en diferentes lugares del mundo, un efecto positivo en los conocimientos sobre la nutrición en el colegio y en algunos casos una mejoría en cuanto a los comportamientos en salud relacionados a la nutrición y la actividad física $^{22-27}$.

\section{Materiales y métodos}

Se realizó una visita exploratoria a las escuelas y se estableció un diálogo con las directivas y maestros acerca de lo que significaba un colegio saludable y las fortalezas y debilidades percibidas por cada uno de ellos para mejorar la salud. Posteriormente, se realizó una evaluación antropométrica. Luego, se obtuvieron los Z score de la talla para la edad y el índice de masa corporal para la edad de acuerdo con los estándares de crecimiento de la Organización Mundial de la Salud $^{7}$, para lo cual se utilizó Epi info 7. Adicionalmente, se realizó la clasificación del índice de masa corporal para la edad de acuerdo a los estándares de crecimiento del CDC.

\section{Grupo evaluador}

El equipo que estandarizó e implementó la prueba estuvo constituido por una médica pediatra, médica $M A, P h D$, dos médicas rurales y un médico interno del programa de responsabilidad social humanitaria Vidas Móviles.

\section{Recolección de la información}

El proceso de la evaluación antropométrica en el colegio $(\mathrm{x})$ fue realizado entre el 4 y el 25 de marzo y en el colegio $(y)$ los días 4 y 5 de abril de 2013.

La evaluación del peso se efectuó con una báscula digital marca "Seca Clara 830" la cual fue calibrada, ubicada sobre una superficie plana y con buen soporte, con todos sus ángulos en contacto con el piso. Los niños fueron dejados con la mínima cantidad de ropa posible y se garantizó que se ubicaran directamente en la mitad de la superficie de la báscula, permaneciendo en posición recta y erguida. Una vez reportado el resultado, se registró el peso exacto obtenido en kilogramos. Posteriormente, se repitió el procedimiento para validar la medida anterior, comparándolo con la primera medición. En los casos en que los valores difirieron dentro de la variabilidad permitida, fueron promediados. De lo contrario se realizó una tercera toma y se promediaron los dos valores que tuvieran la variabilidad permitida entre sí. Toda esta información se recolectó directamente en una agenda electrónica.

En cuanto a la medición de la talla, se ubicó el estadiómetro de acrílico de 2 metros, sobre una superficie plana, 
contra una pared; se situó a cada niño en el estadiómetro, en posición erguida comprobando que la parte de atrás de los talones, las pantorrillas, los glúteos, la espalda y la cabeza tocaran la pared. Los brazos colgaban libremente a los lados del tronco. Finalmente, se revisó que los talones estuvieran juntos y no elevados, las rodillas sin doblar y contra la pared. Previo a la medición, se retiraron los adornos de la cabeza y los zapatos. Se midió la talla utilizando una escuadra formando un ángulo de 90 grados con el punto más alto de la cabeza de los niños. Se registró la talla en metros directamente en una agenda electrónica.

\section{Consideraciones éticas}

Este estudio no tuvo ningún riesgo para los participantes. Los rectores de ambas instituciones autorizaron el uso de los datos para el análisis, respetando la confidencialidad de los mismos.

Las directivas de las instituciones una vez presentado el protocolo, realizaron una socialización con los profesores y los padres de familia quienes en todos los casos autorizaron las evaluaciones a los niños. Solamente, se excluyeron de la muestra los niños que en el día de la recolecciòn de datos no asistieron al colegio. No hubo exclusiones por morbilidad.

\section{Resultados}

Los resultados de este estudio exploratorio están centrados en la descripción de las condiciones antropométricas de dos colegios, cuya muestra fue seleccionada por conveniencia. No se pretendió establecer comparaciones entre los mismos.

\section{Características de la población}

Se obtuvo información sobre las características socioeconómicas de las familias de los niños del colegio $x$ a partir de una encuesta realizada por la institución a los padres de familia. No fue posible obtener dicha información en el otro colegio.

El $96 \%$ de las familias se encuentran clasificadas como nivel 1 por la metodología de clasificación de los beneficiarios del subsidio en salud SISBEN y un $4 \%$ de las familias se encuentran clasificadas como SISBEN 2. El $47 \%$ de las familias declaró ingresos iguales a un salario mínimo legal vigente, el $40 \%$ declaró salarios que oscilan entre uno y dos salarios mínimos legales vigentes y el $21 \%$ un ingreso menor a un salario mínimo legal vigente. El $2 \%$ declaró ingresos mayores a dos salarios mínimos legales vigentes.

En cuanto a la estructura familiar, el $62 \%$ de las familias son nucleares y el $22 \%$ son monoparentales. El $11 \%$ de las familias son extensas y el $5 \%$ recompuestas.

El $41 \%$ de los padres viven en unión libre, el $27 \%$ están separados, el $22 \%$ se encuentran casados. Por otra parte $4 \%$ son madres o padres solteros y un $4 \%$ son viudos.

En cuanto al nivel educativo de los padres, el 52,2\% completó el bachillerato.

El 45\% de las familias cuenta con vivienda propia, algunos de ellos destinan parte de sus ingresos al pago de la cuota de su casa. El $34 \%$ reside en vivienda arrendada y el $18 \%$ reside en vivienda compartida.

\section{Caracterización de los colegios}

En cuanto a la organización de cada uno de los establecimientos escolares, encontramos que en el colegio $x$ existe una escuela de padres, que convoca a las familias de manera continua y permanente para que conozcan los procesos orientados hacia la salud que se están implementando y poder así, reforzar los hábitos saludables en sus hogares, en especial la actividad física y la nutrición adecuada. En cuanto al colegio $Y$ no pudimos acceder al proyecto educativo institucional (PEI), ni convocar a la junta de padres para obtener la información sobre los aspectos anteriormente mencionados.

Encontramos que el colegio x tiene una relación cercana con los padres de familia, creando espacios como la escuela saludable, en la que se tienen las puertas abiertas en horarios establecidos y reconocidos por la comunidad.

En el colegio $Y$ existe la intención de mantener un contacto con las familias, pero para el equipo no fue clara la metodología utilizada, ni los horarios establecidos.

No existe ningún tipo de programa de alimentación escolar en ninguna de las dos instituciones para promover hábitos de alimentación saludable. La salud no se encuentra incluida como dimensión transversal en el proyecto educativo institucional del colegio $\mathrm{x}$; solo existe una orientación a transformarse en una escuela saludable.

En ambos colegios existen tiendas escolares. En la tienda del colegio $x$, ofrecen principalmente: alimentos procesados, seguidos por carbohidratos complejos freídos $\mathrm{u}$ horneados y azúcares refinados. Ocasionalmente, en la tienda escolar hay disponibilidad de frutas. En el colegio $\mathrm{Y}$, ofrecen principalmente: azúcares refinados, seguidos por alimentos compuestos por carbohidratos complejos freídos $\mathrm{u}$ horneados, alimentos procesados $\mathrm{y}$ frutas.

\section{Evaluación antropométrica}

Se realizó una evaluación antropométrica a 887 niños y niñas de los cuales $54,9 \%$ correspondían al colegio x y $45,1 \%$ correspondían al colegio Y. (En la jornada de la mañana). Los estadísticos descriptivos del total de los estudiantes se encuentran en la tabla 1 y en la tabla 2.

En cuanto a la distribución por sexo, hay un ligero predominio del sexo masculino en ambos colegios, principalmente en el colegio $x$.

La mayoría de los estudiantes presentan un índice de masa corporal para la edad adecuado, no obstante, el $16,41 \%$ se encontraban clasificados con obesidad o sobrepeso (fig. 1), siendo más alto este porcentaje en el colegio Y que en el colegio x (fig. 2).

Hay mayor prevalencia de: obesidad, sobrepeso, riesgo para delgadez y delgadez en los niños que en las niñas (fig. 3). La anterior observación ocurre también en cada uno de los colegios por separado (figs. 4 y 5).

Se calculó el percentil al cual pertenecía cada estudiante de acuerdo a los estándares de crecimiento del CDC y se observó que cinco estudiantes se encontraban por encima del percentil 99. Como se mencionó previamente se ha encontrado que por encima de dicho percentil se encuentran anomalías en la bioquímica sanguínea y un mayor riesgo 
Tabla 1 Estadísticos descriptivos de los estudiantes de ambos colegios

\begin{tabular}{|c|c|c|c|c|c|c|c|}
\hline \multicolumn{8}{|c|}{ Índice de Masa Corporal } \\
\hline Edad & Obs & Promedio & Desviación Estándar & Mínimo & Mediana & Máximo & Moda \\
\hline 5 años & 74 & 15,3932 & 1,3354 & 12,15 & 15,43 & 21,46 & 15,49 \\
\hline 6 años & 121 & 15,3474 & 1,4615 & 11,69 & 15,15 & 21,36 & 15,74 \\
\hline 7 años & 145 & 15,7336 & 1,7124 & 12,73 & 15,53 & 22,83 & 14,24 \\
\hline 8 años & 156 & 16,4027 & 2,1738 & 13,14 & 16,02 & 25,94 & 16,39 \\
\hline 9 años & 152 & 16,2637 & 2,1562 & 12,95 & 15,875 & 25,7 & 14,35 \\
\hline 10 años & 119 & 16,7625 & 2,24 & 12,94 & 16,34 & 23,96 & 15,38 \\
\hline 11 años & 81 & 17,4619 & 2,376 & 13,56 & 17,26 & 26,34 & 15,89 \\
\hline 12 años & 29 & 17,3048 & 2,2958 & 12,98 & 17,03 & 21,73 & 17,12 \\
\hline 13 años & 8 & 21,0538 & 4,1211 & 15,62 & 23,005 & 25,64 & 15,62 \\
\hline 14 años & 1 & 17,79 & NeuN & 17,79 & 17,79 & 17,79 & 17,79 \\
\hline \multicolumn{8}{|c|}{ Peso } \\
\hline Edad & Obs & Promedio & Desviación Estándar & Mínimo & Mediana & Máximo & Moda \\
\hline 5 años & 74 & 17,6743 & 1,9982 & 15 & 17,3 & 25,5 & 18 \\
\hline 6 años & 121 & 19,0909 & 2,4131 & 14,6 & 19,1 & 28,5 & 19,4 \\
\hline 7 años & 145 & 21,6931 & 3,3049 & 16,3 & 21 & 33,8 & 21,7 \\
\hline 8 años & 156 & 24,7013 & 4,357 & 17,3 & 23,55 & 38,7 & 26,3 \\
\hline 9 años & 152 & 26,848 & 4,9721 & 18,2 & 26 & 45,3 & 22,5 \\
\hline 10 años & 119 & 29,7765 & 5,2998 & 19,7 & 29,2 & 49,9 & 29,2 \\
\hline 11 años & 81 & 33 & 5,6067 & 22,7 & 31,9 & 50,9 & 26,1 \\
\hline 12 años & 29 & 34,8172 & 5,735 & 24,1 & 33,2 & 49 & 30,7 \\
\hline 13 años & 8 & 46,375 & 14,1184 & 27,6 & 50,1 & 63,2 & 27,6 \\
\hline 14 años & 1 & 45,1 & NeuN & 45,1 & 45,1 & 45,1 & 45,1 \\
\hline \multicolumn{8}{|c|}{ Talla } \\
\hline Edad & Obs & Promedio & Desviación Estándar & Mínimo & Mediana & Máximo & Moda \\
\hline 5 años & 74 & 107,0946 & 3,8089 & 98,5 & 106,85 & 118,1 & 109 \\
\hline 6 años & 121 & 111,4215 & 3,887 & 100,2 & 111,5 & 121,6 & 111 \\
\hline 7 años & 145 & 117,2024 & 4,5724 & 107,5 & 116,6 & 128 & 114 \\
\hline 8 años & 156 & 122,4346 & 5,1295 & 107 & 122,5 & 133 & 119 \\
\hline 9 años & 152 & 128,1171 & 5,8322 & 113 & 128 & 145 & 126 \\
\hline 10 años & 119 & 133,02 & 5,78 & 117,3 & 133,4 & 151 & 134 \\
\hline 12 años & 29 & 141,69 & 6,54 & 130,1 & 141,3 & 157,7 & 134 \\
\hline 13 años & 8 & 146,74 & 10,31 & 131 & 147,15 & 158,5 & 131 \\
\hline 14 años & 1 & 159,2 & NeuN & 159,2 & 159,2 & 159,2 & 159,2 \\
\hline
\end{tabular}

Tabla 2 Distribución por sexo de la población de ambos colegios

\begin{tabular}{|c|c|c|c|c|}
\hline Sexo & Frecuencia & & Porcentaje & Total \\
\hline Masculino & 458 & & $51,69 \%$ & \\
\hline Femenino & 428 & & $48,31 \%$ & 886 \\
\hline Y Frecuencia & Y Porcentaje & Y Total & & Porcentaje de la muestra \\
\hline 200 & $50,38 \%$ & \multirow{2}{*}{\multicolumn{2}{|c|}{397}} & \multirow{2}{*}{$44,80 \%$} \\
\hline 197 & $49,62 \%$ & & & \\
\hline x Frecuencia & x Porcentaje & \multicolumn{2}{|l|}{ x Total } & Porcentaje de la muestra \\
\hline 258 & $52,76 \%$ & \multirow{2}{*}{\multicolumn{2}{|c|}{489}} & \multirow[b]{2}{*}{$55,00 \%$} \\
\hline 231 & $47,24 \%$ & & & \\
\hline
\end{tabular}




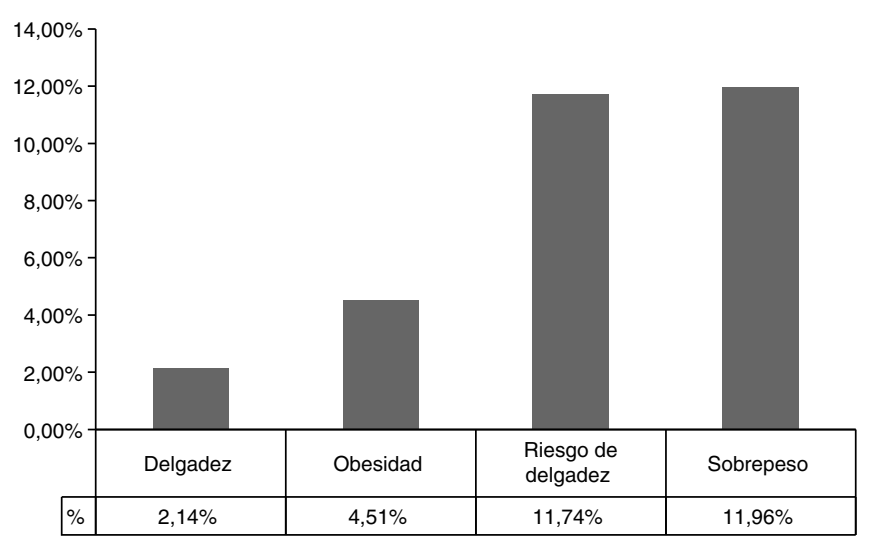

Figura 1 Índice de masa corporal para la edad en el total de la población analizada.

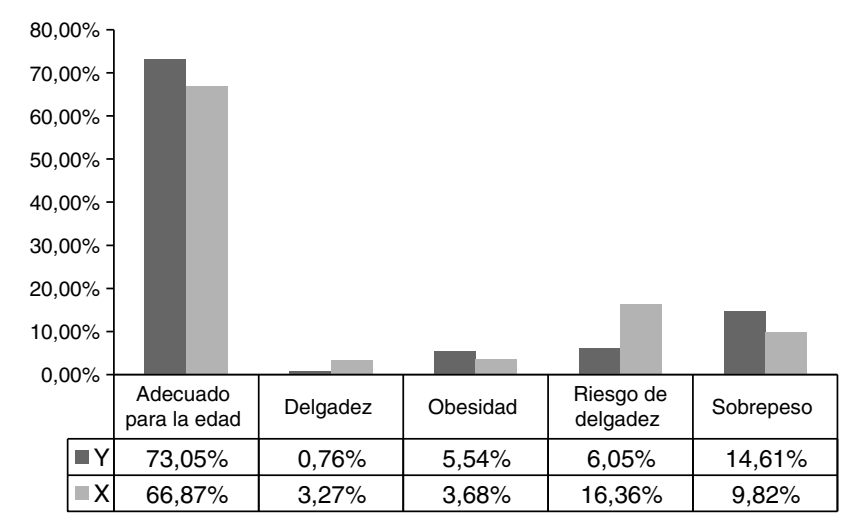

Figura 2 Índice de Masa corporal para la edad en cada uno de los colegios.



Figura 3 Índice de masa corporal para la edad de acuerdo al sexo.

de obesidad severa en la adultez ${ }^{9}$. De los estudiantes que se encontraban por encima del percentil 99, cuatro eran hombres y cuatro de ellos pertenecían al colegio $\mathrm{Y}$, dos de ellos tenían ocho años y los otros tenían 4, 5 y 6 años, respectivamente.

Para hablar sobre la salud en la escuela, se realizó un grupo focal, que fue grabado con autorización de los participantes quienes correspondían a 9 profesores del colegio $x$ encargados de la docencia en los cursos de transición a quinto de primaria. La grabación fue transcrita con mínima

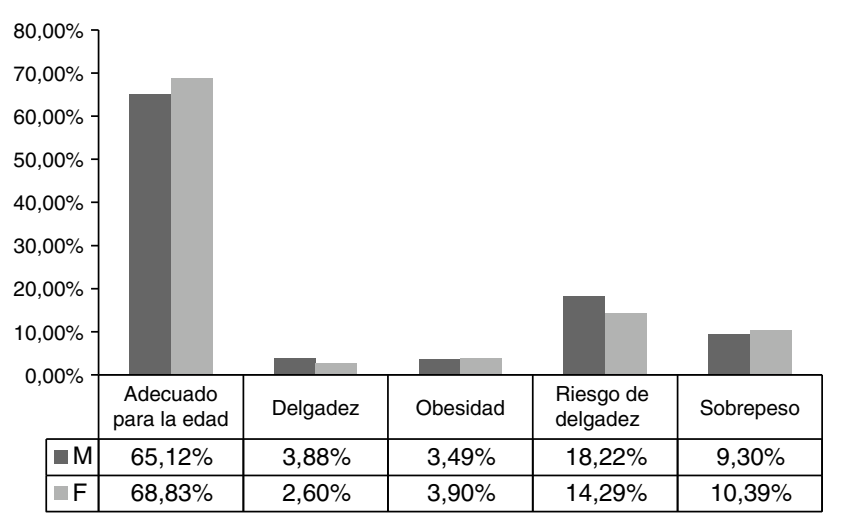

Figura 4 Índice de masa corporal para la edad de acuerdo al sexo en el colegio $x$.

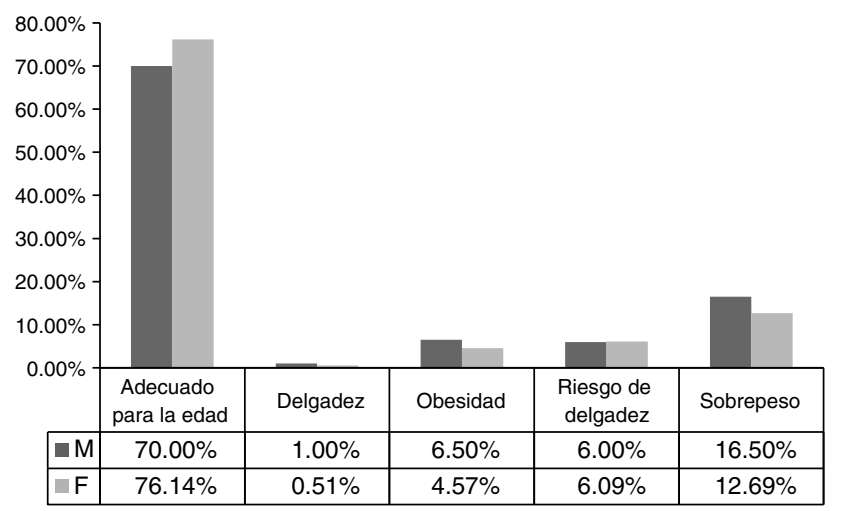

Figura 5 Índice de masa corporal para la edad de acuerdo al sexo en el colegio $\mathrm{Y}$.

edición. A partir del texto transcrito, de forma manual se identificaron las categorías emergentes sobre la noción de "escuela saludable ideal", las cuales se agruparon en un diagrama (Diagrama 1).

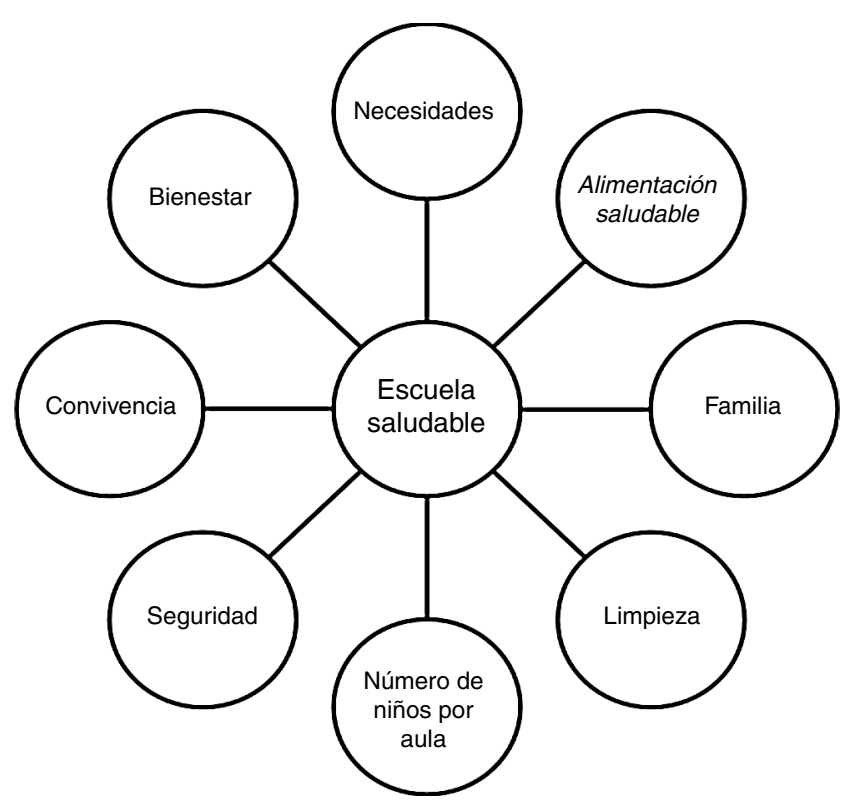

Diagrama 1 Categorías encontradas en el grupo focal realizado con los profesores sobre una Escuela Saludable Ideal. 


\section{Discusión}

En la valoración antropométrica se encontraron diferencias entre ambos colegios en cuanto a la prevalencia del sobrepeso y la obesidad clasificados de acuerdo con el índice de masa corporal para la edad. Se requiere mayor investigación para determinar si estas diferencias son significativas y para determinar los posibles factores asociados.

Los anteriores resultados se diferenciaron por el sexo, evidenciando una menor prevalencia de la obesidad y el sobrepeso en las niñas que en los niños. Consideramos necesario continuar profundizando para conocer las causas de estas diferencias.

Aunque la alimentación saludable fue incluida dentro de las categorías relevantes para los participantes en el grupo focal, en su proceso de transformación hacia una escuela saludable, esta percepción no se vio reflejada en el proyecto educativo institucional analizado, ni en las medidas tomadas por parte de los colegios para mejorar la alimentación, luego de conocer los resultados. Llama la atención que la actividad física no fuera una categoría que surgiera como importante en las conclusiones del grupo focal.

Es de resaltar que la estrategia de involucrar a las familias y vincularlas a través de las actividades curriculares, incluida en las categorías que los profesores consideran relevantes dentro de la transformación hacia una escuela saludable, podría ser una iniciativa adecuada para favorecer el inicio de unos hábitos alimenticios saludables que podrían facilitar un mejor estado nutricional de los niños en un futuro cercano 28,29 .

\section{Conclusiones}

Esta primera exploración de la situación nutricional de los niños en edad escolar en los colegios de Ciudad Bolívar invita a pensar en la necesidad de realizar una nueva investigación que nos permita establecer las asociaciones causales entre los resultados de la valoración antropométrica y las condiciones estructurales y de vida en los colegios, las comunidades y las familias. Explorar si ocurren consecuencias diferenciales por la alimentación entre: colegios, localidades, espacios urbanos, rurales, y por edad y sexo.

Las aparentes diferencias en cuanto a la prevalencia de desnutrición en los colegios, plantea la necesidad de una mayor investigación que permita conocer el impacto de las estrategias nutricionales ofrecidas por los diferentes actores educativos en las zonas vulnerables de la ciudad con el fin de encaminarlas hacia un diseño de escuelas saludables con planes alimentarios y de actividad física que promuevan modos de vida más preventivos de enfermedades crónicas no transmisibles en otros cursos de la vida.

\section{Reconocimientos y agradecimientos}

Queremos agradecer a los estudiantes de medicina y médicos en servicio social obligatorio quienes contribuyeron en este estudio. A los maestros y directivas de los colegios que facilitaron la infraestructura y animaron nuestra labor.

\section{Responsabilidades éticas}

Protección de personas y animales. Los autores declaran que para esta investigación no se han realizado experimentos en seres humanos ni en animales.

Confidencialidad de los datos. Los autores declaran que en este artículo no aparecen datos de pacientes.

Derecho a la privacidad y consentimiento informado. Los autores declaran que en este artículo no aparecen datos de pacientes.

\section{Financiación}

Este estudio fue realizado con los recursos del programa de responsabilidad social universitaria Vidas Móviles de la Pontificia Universidad Javeriana de Bogotá

\section{Conflicto de intereses}

Los autores declaran no tener conflictos de interés.

\section{Bibliografía}

1. Borrero García Camilo. Derechos Humanos. Ideas y dilemas para animar su comprensión. Bogotá, Colombia: Fundación Centro de Investigación y Educación Popular. En publicación.

2. Kasper NM, Herrán OF. Obesity prevalence in Colombian adults is increasing fastest in lower socioeconomic status groups and urban residents: Results from two nationally representative surveys. Public Health Nutr. 2014;17:2398-406.

3. Lissau I, Moreno LA, Pietrobelli A, Widhalm K. New insights into the field of children and adolescents' obesity: the European perspective. Int J Obes Relat Metab Disord. 2004;10:1189-96.

4. Neovius M, Linné Y, Barkeling B, Rössner S. Discrepancies between classification systems of childhood obesity. Obes Rev. 2004; 5:105-14.

5. Cole TJ, Bellizzi MC, Flegal KM, Dietz WH. Establishing a standard definition for child overweight and obesisty worldwide: International survey. Br Med J. 2000:320.

6. Juonala M, Raitakari M, Vikari Jorma SA, Raitakari OT. Obesity in Children is not an independent predictor of carotid IMT in adulthood: The Cardiovascular Risk in Young Finns Study. Atherosclerosis. 2005; 185:388-93.

7. Lloyd LJ, Langley- Evans SC, McMullen S. Childhood obesity and adult cardiovacular disease risk: a systematic review. Int J Obes. 2009;34:18-28.

8. Juonala M, Magnussen CG, Berenson GS, Venn A, Burns TL, Sabin MA, et al. Childhood adiposity, adult adiposity, and cardiovascular risk factors. N Engl J Med. 2011;365:1876-85.

9. Freedman DS, Mei Z SS, Berenson GS, Dietz WH. Cardiovascular Risk factors and Excess adiposity among overweight Children and adolescents: The Bogalusa heart Study. J Pediatr. 150:12-7.

10. Shashaj B, Bedogni G, Graziani MP, Tozzi AE, DiCorpo ML, Morano D, Tacconi L, Veronelli P, Contoli B. Origin of cardiovascular Risk in Overweight Preschool Children: a Cohot study of cardiometabolic risk at the onset of obesity. JAMA Pediatr. 2010;168.

11. Weiss R, Dziura J, Burgert TS, Tamborlane WV, Taksali SE, Yeckel $\mathrm{CW}$, et al. Obesity and the metabolic syndrome in children and adolescents. N Engl J Med. 2004;350:2362-74.

12. Hannon TS, Rao G, Arslanian SA. Childhood obesity and type 2 diabetes mellitus. Pediatrics. 2005;116:473-80. 
13. Meizi He. Obesogenic neighbouhoods: the impact of neighbourhood restaurants an convenience stores on adolescents food consumption behaviours. Public Health Nutr. 2012;15:2331-9.

14. Virtaten M. Fast- Food outlets and grocery stores near shcool and adolescents eating habits and overweight in Finland. Eur J Public Health. 2015:25.

15. Hilmers A, Hilmers DC, Dave J. Neighborhood disparities in access to healthy foods and their effects on environmental justice. Am J Public Health. 2012;102:1644-54.

16. Hospital Vista Hermosa, Secretario Distrital de Salud, Alcalde Mayor de Bogotá. Diagnóstico Local con Participación Social en Salud Ciudad Bolívar [Internet]. Alcalde Mayor de Bogotá; 2011. Available from: http://www.hospitalvistahermosa.gov.co/ web/node/sites/default/files/boletines_2012/DIAGNOSTICO_ LOCAL_2011_act.pdf

17. Hospital Vista Hermosa, Secretario Distrital de Salud, Alcalde Mayor de Bogotá. Sistema de Vigilancia Nutricional y Alimentaria- SISVAN [Internet]. 2011. Available from: http://www.hospitalvistahermosa.gov.co/web/node/sites/ default/files/boletines_2012/COVE/JULIO/SISVAN.pdf

18. Ashe LM, Sonnino R. At the crossroads: new paradigms of food security, public health nutrition and school food. Public Health Nutr. 2013;16:1020-7.

19. Nichols P, Ussery-Hall A, Griffin-Blake S, Easton A. The Evolution of the Steps Program, 2003-2010: Transforming the Federal Public Health Practice of Chronic Disease Prevention. Prev Chronic Dis [Internet]. 2012 Feb 2 [cited 2015 Jan 2];9. Available from: http://www.ncbi.nlm.nih.gov/ pmc/articles/PMC3340214/

20. World Health Organization. What is a health promoting school? [Internet]. WHO. 2014 [cited 2015 Jan 6]. Available from: http://www.who.int/school_youth_health/gshi/hps/en/

21. Langford R, Bonell CP, Jones HE, Pouliou T, Murphy SM, Waters E, et al. The WHO Health Promoting School framework for improving the health and well-being of students and their academic achievement. In: The Cochrane Collaboration, editor. Cochrane Database of Systematic Reviews [Internet]. Chichester, UK:
John Wiley \& Sons, Ltd; 2014 [cited 2015 Jan 2]. Available from: http://pesquisa.bvsalud.org/portal/resource/es/mdl24737131

22. Lobos Fernández LL, Leyton Dinamarca B, Kain Bercovich J, Vio del Río F. [Evaluation of an education intervention for childhood obesity prevention in basic schools in Chile]. Nutr Hosp. 2013;28:1156-64.

23. Prelip M, Slusser W, Thai CL, Kinsler J, Erausquin JT. Effects of a school-based nutrition program diffused throughout a large urban community on attitudes, beliefs, and behaviors related to fruit and vegetable consumption. J Sch Health. 2011;81:520-9.

24. Shepherd J, Harden A, Rees R, Brunton G, Garcia J, Oliver $\mathrm{S}$, et al. Young people and healthy eating: a systematic review of research on barriers and facilitators. Health Educ Res. 2006;21:239-57.

25. Tarro L, Llauradó E, Albaladejo R, Moriña D, Arija V, Solà R, et al. A primary-school-based study to reduce the prevalence of childhood obesity-the EdAl (Educació en Alimentació) study: a randomized controlled trial. Trials. 2014;15:58.

26. Te Velde SJ, Lennert Veerman J, Tak NI, Bosmans JE, Klepp K-I, Brug J. Modeling the long term health outcomes and cost-effectiveness of two interventions promoting fruit and vegetable intake among schoolchildren. Econ Hum Biol. 2011;9:14-22.

27. Wilson DB, Jones RM, McClish D, Westerberg AL, Danish S. Fruit and vegetable intake among rural youth following a schoolbased randomized controlled trial. Prev Med. 2012;54:150-6.

28. Attorp A, Scott JE, Yew AC, Rhodes RE, Barr SI, Naylor PJ. Associations between socioeconomic, parental and home environment factors and fruit and vegetable consumption of children in grades five and six in British Columbia, Canada. BMC Public Health. 2014;14:150.

29. Østbye T, Malhotra R, Stroo M, Lovelady C, Brouwer R, Zuc$\operatorname{ker} \mathrm{N}$, et al. The effect of the home environment on physical activity and dietary intake in preschool children. Int $\mathrm{J}$ Obes. 2013;37:1314-21. 